\title{
A test of equivalence
}

Phys. Rev. Lett. (in the press); preprint available at https://arxiv.org/abs/1902.04193

In Einstein's theory of general relativity (GR), the equivalence principle can be broken down to the sum of three main principles: the weak equivalence principle, the local position invariance (LPI) and the local Lorentz invariance. The GRAVITY collaboration uses observations of the star S2 close to Sgr $A^{\star}$ - a mere $\sim 16$ light hours away at its closest - to constrain the LPI in a regime previously unattainable with other experiments.

The supermassive black hole Sgr $A^{*}$ at the centre of the Milky Way with test-mass S2 in orbit around it makes an ideal laboratory to test GR. Having already previously established that S2 follows the orbit predicted by GR, the GRAVITY collaboration now shows that local nongravitational measurements are independent of their location in spacetime. To constrain this spacetime invariance, the frequencies of two different absorption lines in the spectrum of S2 (He I and Bry) are studied as a function of the position of S2 with respect to Sgr $\mathrm{A}^{\star}$ and therefore of the gravitational potential experienced by S2.

Based on this comparison, the GRAVITY collaboration manages to place an upper limit to any violation of the LPI of $\Delta \beta$ $\leq 5 \times 10^{-2}$, consistent with zero given the uncertainties. While Earth-bound experiments have placed upper limits to $\Delta \beta$ with significantly higher precision, this experiment does so for a gravitational field that is several orders of magnitude stronger than most previous experiments. With the Extremely Large Telescope operational by the next pericentre passage of $\$ 2$, new measurements will improve this upper limit by up to four orders of magnitude.

Marios Karouzos

Published online: 12 March 2019

https://doi.org/10.1038/s41550-019-0742-y 